\title{
The effect of maternal forced exercise on offspring pain perception, motor activity and anxiety disorder: the role of 5-HT2 and D2 receptors and CREB gene expression
}

\author{
Ozra Motaghinejad', Majid Motaghinejad ${ }^{1, *}$, Manijeh Motevalian', Fatemeh Rahimi-Sharbaf ${ }^{2}$, Tabassom Beiranvand ${ }^{1}$ \\ ${ }^{1}$ Razi Drug Research Center and Department of Pharmacology, School of Medicine, Iran University of Medical Sciences, Tehran, Iran \\ ${ }^{2}$ Department of Gynecology, Tehran University of Medical Sciences, Tehran, Iran
}

The effect of maternal forced exercise on central disorders in offsprings has been shown but the mechanism is still unclear. In this study, the role of $5-\mathrm{HT}_{2}$ and $\mathrm{D}_{2}$ receptors in neuroprotective effects of maternal forced exercise on offspring neurodevelopment and neurobehavioral symptoms is evaluated. Sixty pregnant rats were trained by forced exercise and some behavioral and molecular aspects in their offspring were evaluated in presence of $5-\mathrm{HT}_{2}$ and $\mathrm{D}_{2}$ receptors agonists and antagonists. The results showed that maternal forced exercise causes increase of pain tolerability and increase latency of pain perception in offspring in hot plate test, writhing test and tail flick test. Also maternal forced exercise causes decrease of depression and anxiety like behavior in offsprings. On the other hand, treatment of mothers by forced ex- ercise in combination with $5-\mathrm{HT}_{2}$ and $\mathrm{D}_{2}$ receptor antagonists inhibited the protective effects of forced exercise and cause disturbance in pain perception and tolerability and increase depression and anxiety in offsprings. Also expression of cyclic AMP response element binding protein (CREB) was changed in all experimental groups. In conclusion, our data suggested that maternal forced exercise causes neurobehavioral protective effect on offsprings and this effect might probably be mediated by $5-\mathrm{HT}_{2}$ and $\mathrm{D}_{2}$ receptors and activation of CREB gene expression.

Keywords: Maternal forced exercise, Pain perception, Motor activity, Anxiety, Depression

\section{INTRODUCTION}

Several studies demonstrated that exercise lowers the stress and anxiety and increases endorphin secretion in brain (Salmon, 2001). Physical activity improves symptoms of anxiety in healthy population and patients; chronic forced exercise shows anxiolytic-like effects in some experiments (Lawlor and Hopker, 2001). It has also been shown that exercise can counteract depression and can increase the cognitive activity (Kim and Seo, 2013; Kramer et al., 2006; Tomporowski, 2003). Chronic exercise in mice resulted in antidepressant-like behavioral changes, that may involve a brain-derived neurotrophic factor (BDNF) related mechanism similar to what is hypothesized for antidepressant drug treatment (Cotman and Berchtold, 2002; Park et al., 2013). Exercise in- creases synthesis and release of dopamine, stimulates neuroplasticity and promotes feelings of well-being (Cotman and Berchtold, 2002; Motaghinejad et al., 2014b; Ojoawo et al., 2017). Many previous studies have shown that maternal exercise enhances neurogenesis in rat's pups but the exact mechanism of this effect remains unclear (Lee et al., 2006; Kim et al., 2007; Kim, 2013). Maternal treadmill running during pregnancy has beneficial effects on short-term memory, hippocampal cell survival and the expression of BDNF mRNAs in rat pups (Aksu et al., 2012; Kim et al., 2017; Uysal et al., 2011). On the other hand, many neurotransmitters and neuromodulators can have effect on neurogenesis in fetus during development (Salmon, 2001). Some other studies have shown that dopamine and serotonin increase the neurogenesis and activate the neurogenesis pathways via activation of
${ }^{*}$ Corresponding author: Majid Motaghinejad (D) https://orcid.org/0000-0003-3449-4224 Razi Drug Research Center \& Department of Pharmacology, School of Medicine, Iran University of Medical Sciences, Tehran 14155-6183, Iran

Tel: +98-9391974237, Fax: +98-2188622696, E-mail: dr.motaghinejad6@gmail.com Received: May 20, 2017 / Accepted: September 13, 2017
This is an Open Access article distributed under the terms of the Creative Commons Attribution Non-Commercial License (http://creativecommons.org/licenses/by-nc/4.0/) which permits unrestricted non-commercial use, distribution, and reproduction in any medium, provided the original work is properly cited. 
$\mathrm{D}_{2}$ and 5-HT $\mathrm{HT}_{2}$ receptors respectively (Benninghoff et al., 2012; Gaspar et al., 2003; Whitaker-Azmitia, 2001). Moreover, research studies have shown that cyclic AMP response element binding protein (CREB) acts as major transcription factor in brain neurodevelopment and neurogenesis (Cho et al., 2013; Dworkin and Mantamadiotis, 2010; Gass and Riva, 2007; Merz et al., 2011). Therefore, the aim of the present study is the evaluation of the influence of two neurotransmitters, dopamine and serotonin, and their receptors, $\mathrm{D}_{2}$ and 5- $\mathrm{HT}_{2}$, on maternal forced exercise protective effect on offspring neurodevelopment and neurobehavioral signs; such as pain perception, anxiety, depression and also the involvement of CREB in this type of development was evaluated.

\section{MATERIALS AND METHODS}

\section{Animal}

Sixty-eight pregnant Wistar rats, weighting $250 \mathrm{~g}$, were used for all the experiments. They were divided randomly into 10 groups as mentioned bellow. After delivery, their offspring's were kept in the light controlled room under a 12-hr light and dark cycle with temperature of $22^{\circ} \mathrm{C} \pm 2^{\circ} \mathrm{C}$. Food and water were available ad libitum. Before the tests starts, the animals were allowed to adapt themselves to the laboratory for at least $2 \mathrm{hr}$ and they were used only once. All experimental procedures followed the Guidelines on Ethical Standards for investigation of experimental pain in animals (Motaghinejad et al., 2014b; Motaghinejad et al., 2015a; Noori et al., 2014; Shojaii et al., 2015).

\section{Drug}

Haloperidol, trazodone, bromocriptine, and ketanserin were purchased from Sigma-Aldrich Inc. (St. Louis, MO, USA).

\section{Experimental design of pregnant rats}

All pregnant animals were divided randomly into 10 groups (6 rats in each group).

- As the negative control, group 1 received normal saline $(0.2$ $\mathrm{mL} /$ rat. intraperitoneally, once daily) during their pregnancy.

- Group 2 were trained with forced exercise only during their pregnancy.

- Group 3 were treated with haloperidol $(5 \mathrm{mg} / \mathrm{kg}$, intraperitoneally, once daily) in combination with forced exercise during their pregnancy.

- Group 4 were treated with bromocriptine $(10 \mathrm{mg} / \mathrm{kg}$, intraperitoneally, once daily) in combination with forced exercise during their pregnancy.
- Group 5 were treated with O-4310 (10 mg/kg, intraperitoneally, once daily) in combination with forced exercise during pregnancy

- Group 6 were treated with trazodone $(5 \mathrm{mg} / \mathrm{kg}$, intraperitoneally, once daily) in combination with forced exercise during their pregnancy.

- Group 7 were treated with haloperidol $(5 \mathrm{mg} / \mathrm{kg}$, intraperitoneally, once daily) during their pregnancy.

- Group 8 were treated with bromocriptine $(10 \mathrm{mg} / \mathrm{kg}$, intraperitoneally, once daily) during their pregnancy.

- Group 9 were treated with trazodone $(5 \mathrm{mg} / \mathrm{kg}$, intraperitoneally, once daily) during their pregnancy.

- Group 10 were treated with O-4310 (10 mg/kg, intraperitoneally, once daily) during their pregnancy.

\section{Treadmill forced exercise protocol}

Rats were allowed to run on a motor-driven leveled treadmill (Model T408E, Diagnostic \& Research Instruments Co., Taoyuan, Taiwan). The animals of groups 2, 3, and 4 were trained with treadmill for $30 \mathrm{~min} /$ day, 5 days per week. The training speed was $10 \mathrm{~m}$ per minute (for the first week) and increased to 20 and 30 $\mathrm{m}$ per minute in the second and the third week respectively. The slope and the intensity of the exercise were settled as $0^{\circ}$ at the first $10 \mathrm{~min}, 5^{\circ}$ for the second $10 \mathrm{~min}$ and $15^{\circ}$ for the last $25 \mathrm{~min}$ (Motaghinejad et al., 2014b; Motaghinejad et al., 2015b).

\section{Behavioral and molecular assays in offspring}

After the treatment of pregnant rats with the mentioned drugs and the forced exercise, 10 adolescent rats were randomly selected from each group (one offspring rat from each treated mother) and some behavioral and molecular assays were done on offsprings.

\section{Writhing test}

This test is based on the induction of pain by intraperitoneal injection of $10 \mathrm{~mL} / \mathrm{kg}$ acetic acid $(0.8 \%)$. The measurement of severity of the pain was done by counting the number of abdominal contractions known as writhing. The total number of writhings recorded by camera during $30 \mathrm{~min}$ after injection of acetic acid and was counted afterwards. In addition, the onset of the first writhing was recorded as latency time (Motaghinejad et al., 2014a; Shojaii et al., 2015).

\section{Tail flick test}

This test was done in all animals before the treatments. In this test, radiant heat (Tail-flick apparatus model P-131, Pouyaye Ar- 
maghan Co., Tehran, Iran) was applied for assessment of nociception and acute responses in rats. Five millimeters of the tail was submitted to noxious heat $\left(22^{\circ} \mathrm{C} \pm 2^{\circ} \mathrm{C}\right)$. To avoid damaging the tail, if a response did not occur, trial was automatically terminated at 12 sec (cutoff time) (Motaghinejad et al., 2014a; Shojaii et al., 2015).

\section{Hot plate test}

In this test, analgesic activity was measured with a thermostatically heated surface (Hot plate apparatus model P-162, Pouyaye Armaghan Co.) which is maintained at the temperature of $55^{\circ} \mathrm{C} \pm$ $2^{\circ} \mathrm{C}$. The time of reaction was the time that the animal was left on the hot plate until the time the animal licks its hands or jumps up. The onset of the first licking reaction and the duration of the tolerance on the hot plate were recorded as the latency time (Motaghinejad et al., 2014a; Shojaii et al., 2015).

\section{Open field test}

Open field test (OFT) as a standard test was used for the assessment of anxiety and locomotor activity in rodents. To perform this test, an apparatus (Open filed apparatus model P-222, Pouyaye Armaghan Co.) which its bottom was divided into 16 equally spaced squares bordered by opaque high walls of $65.90 \mathrm{~cm}$ was used. All parts of bottom were dyed black except for the $6 \mathrm{~mm}$ broad white lines which divided the ground into 16 squares. During the experiment the whole room except for the open field was kept dark, and the apparatus was illuminated with a $100-\mathrm{W}$ bulb which was focused on the field at the height of about $110 \mathrm{~cm}$ from the ground. For the assessment of anxiety and locomotor activity, each animal was positioned in the center of the test apparatus for a maximum period of 5 min to monitor the following behaviors:

- Ambulation distance: the distance in which the rat crosses the grid lines

- Center square entries: the number of times that the rat crosses one of the central red lines with all its four paws and enters into the central square.

. Center square duration: the time that the rat spends in the central square.

- Rearing: the number of times in which the rat stand on its hind legs in the maze (Motaghinejad et., 2015b).

\section{Elevated plus maze}

Elevated plus maze (EPM) is another test which is used for the anxiety of the experimental animals. EPM equipment (Elevated plus maze apparatus model P-141, Pouyaye Armaghan Co.) in- cludes two opposite arms $(60 \mathrm{~cm} \times 20 \mathrm{~cm})$, which are joined with a central square $(10 \mathrm{~cm} \times 10 \mathrm{~cm})$, forming a plus sign. Two arms are kept open, while the other arms are enclosed with $40 \mathrm{~cm}$ elevated wall. All parts of apparatus are being set in $50-\mathrm{cm}$ height above the ground. All subjects were situated individually in the center of the maze in front of one of the enclosed arms. The time that the animal spent on the open arms were recorded during 5 min for each rat. The time rats spent in open arm was considered as nondepressive behavior (Motaghinejad et al., 2015c).

\section{Quantitative real-time reverse transcription-polymerase chain reaction studies}

After behavioral assay, hippocampus was isolated and total RNA was extracted by using Trizole reagent (Biobasic, Canada) and then treated with DNase I, RNase-Free (Thermo Scientific, Fermentas, Walthman, MA, USA) according to the manufacturer's recommendations. Briefly, in the presence of $0.5-\mu \mathrm{L}$ oligod T primer and Random 6 mers, and reverse transcription at $37^{\circ} \mathrm{C}, 15$ min in $2 \mu \mathrm{L}$ of $5 \mathrm{x}$ PrimeScript buffer (PrimeScriptTM RT reagent kit, Takara, Tokyo, Japan), the total RNA $(0.5 \mu \mathrm{g})$ were denatured. Inactivation of the enzyme $\left(85^{\circ} \mathrm{C}, 5 \mathrm{sec}\right)$ was followed by glyceraldehyde-3-phosphatedehydrogenase (GAPDH) polymerase chain reaction to assess the quality of the template DNA.

The reverse transcription-polymerase chain reaction was carried out with SYBR Premix Ex TaqTM II (TliR-NaseH Plus), Bulk (Takara) kit. The setup of cycling parameters were $95^{\circ} \mathrm{C}$ for $30 \mathrm{sec}$, 42 cycles at $95^{\circ} \mathrm{C}$ for $10 \mathrm{sec}$ and $60^{\circ} \mathrm{C}$ for $32 \mathrm{sec}$ (Dorak, 2007).

The primers for rats GAPDH and CREB (Cinna Gen, Iran):

- GAPDH: Sense: AGACAGCCGCATCTTCTTGT;

Antisense: CCGTTCACACCGACCTTCA

. CREB: sense: CAGACAACCAGCAGAGTGGA;

\section{Antisense: CTGGACTGTCTGCCCATTG}

Samples were analyzed on a real-time qPCR cycler (CFX96, Biorad, Hercules, CA, USA) and the relative mRNA expression of the CREB was normalized to GAPDH expression using the simplified comparative threshold cycle $2^{-\Delta \Delta \mathrm{Ct}}$ method (Dorak, 2007).

\section{Hematoxylin and eosin staining}

For determination of the changes in hippocampal cell density, its isolated tissues were fixed in formalin solution for $48 \mathrm{hr}$ after being washed in physiologic serum. They were dehydrated through graded concentrations of ethanol, embedded in paraffin wax, sectioned in 5- $\mu \mathrm{m}$ thickness, and stained with Mayer's hematoxylin and eosin (H\&E) (Kumar et al., 2014). 


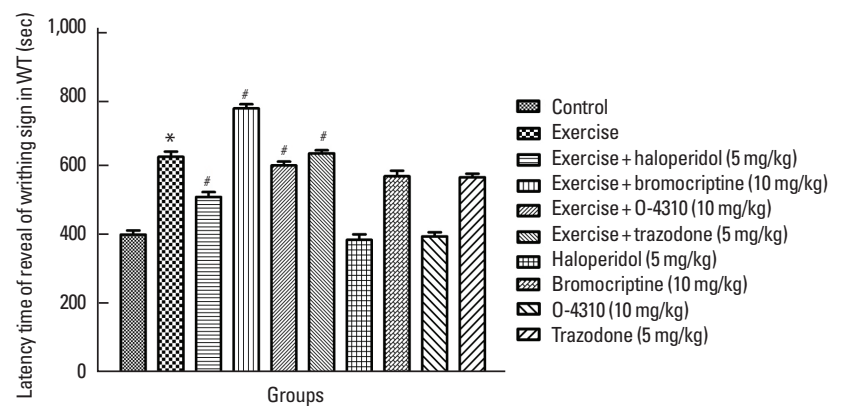

Fig. 1. Latency time for expression of abdominal contraction in writhing test (WT) in the control group and which their mother treated by exercise or its combination with haloperidol, bromocriptine, trazodone or 0-4310 and groups under treatment by haloperidol, bromocriptine, trazodone or 0-4310 alone. Values are presented as mean \pm standard error of the mean $(n=8)$. ${ }^{*} P<0.05$, significant difference from control group. ${ }^{*} P<0.05$, Significant difference from group which their mother were treated by exercise.

\section{Neuropathological examination}

Pathological damage of hippocampal cells in dentate gyrus (DG) region was examined with standard H\&E histological staining of brain paraffinized sections $(5 \mu \mathrm{m})$. The morphological changes and the granular cell density were observed in the DG sector by counting the number of surviving neurons per mm length of the pyramidal layer (Hosseinzadeh et al., 2015; Liu et al., 2006).

\section{Statistical analysis}

All data expressed as the mean \pm standard error of the mean. The differences between positive and negative control groups were analyzed by the unpaired Student $t$-test. The differences between the treatment groups were compared with one-way analysis of variance and then, with a post hoc test of Bonferroni test for groupby-group. The statistically significant value was taken as $P<0.05$.

\section{RESULTS}

\section{The effect of maternal exercise and the role of $D_{2}$ and $5-\mathrm{HT}_{2}$ receptors on offsprings' pain response in WT}

Figs. 1 and 2 indicate the effects of maternal forced exercise on offsprings in writhing test. The maternal forced exercise significantly increased the latency time of writhing sign compared to the control group $(P<0.05)$ (Fig. 1). The treatment of mothers with forced exercise in combination with haloperidol or bromocriptine caused respectively decrease and increase in latency time of writhing sign in comparison with the group which their mothers were treated with the exercise only $(P<0.05)$ (Fig. 1). The treatment of mothers with forced exercise in combination with trazodone or O-4310 respectively increased and decreased

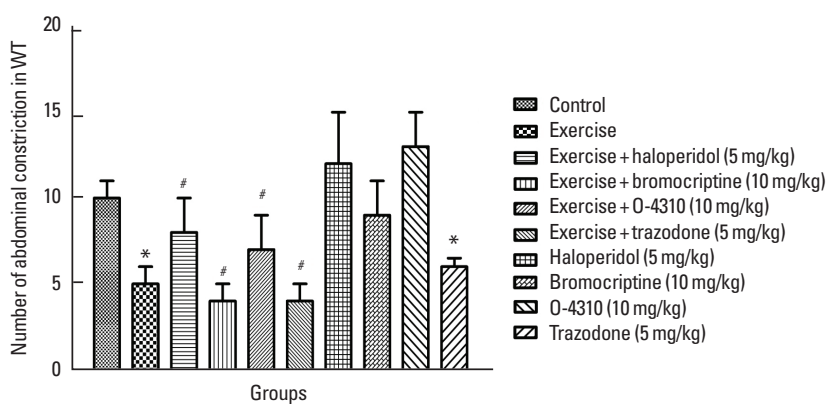

Fig. 2. Number of abdominal contraction in writhing test (WT) in the control group which their mother treated by exercise or its combination with haloperidol, bromocriptine, trazodone or 0-4310 and groups under treatment by haloperidol, bromocriptine, trazodone or 0-4310 alone. Values are presented as mean \pm standard error of the mean $(\mathrm{n}=8)$. ${ }^{*} P<0.05$, significant difference from control group. ${ }^{*} P<0.05$, Significant difference from group which their mother were treated by exercise.

the latency time of writhing sign in comparison with the group whose mothers were treated with exercise only $(P<0.05)$ (Fig. 1). Also, the maternal treatment with bromocriptine or trazodone caused the increase of latency time but the treatment with haloperidol or $\mathrm{O}-4310$ decreased it comparing to the control group but these changes were not statistically significant (Fig. 1). On the other hand, the maternal forced exercise caused significant decrease of number of writhing in comparison to the control group $(P<0.05)$ (Fig. 2). Treatment of mothers by forced exercise in combination with trazodone or bromocriptine significantly increased the number of writhing in comparison with the group which their mothers were treated with exercise only $(P<0.05)$ (Fig. 2). The treatment of mothers with forced exercise in combination with haloperidol or O-4310 significantly decreased the number of writhing in comparison to the group whose mothers were treated with the exercise only $(P<0.05)$ (Fig. 2).

The treatment of mothers with haloperidol or O- 4310 significantly increased the writhing number but the treatment with bromocrptine or trazodone significantly decreased the writhing test in comparison with the control group in which this decrease was significant in trazodone treated group by $P<0.05$ but it was not statistically significant in haloperidol, O-4310 and bromocrptine treated groups (Fig. 2).

\section{The effect of maternal forced exercise and the role of $D_{2}$ and $5-\mathrm{HT}_{2}$ receptors on offsprings' pain response in TFT}

The result of present study indicates that maternal forced exercise significantly increased the latency time in tail flick test (TFT) reaction comparing to the control group $(P<0.05)$ (Fig. 3). In addition, the treatment of mothers by forced exercise in combina- 


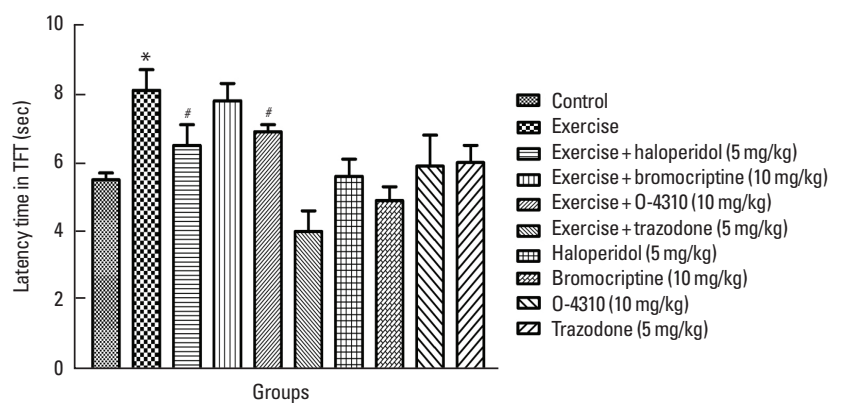

Fig. 3. Latency time of tail flick test (TFT) reaction in the control group and groups which their mother treated by exercise or its combination with haloperidol, bromocriptine, trazodone or 0-4310 and groups under treatment by haloperidol, bromocriptine, trazodone or 0-4310 alone. Values are presented as mean \pm standard error of the mean $(n=8) .{ }^{*} P<0.05$, significant difference from control group. ${ }^{\sharp} P<0.05$, Significant difference from group which their mother were treated by exercise.

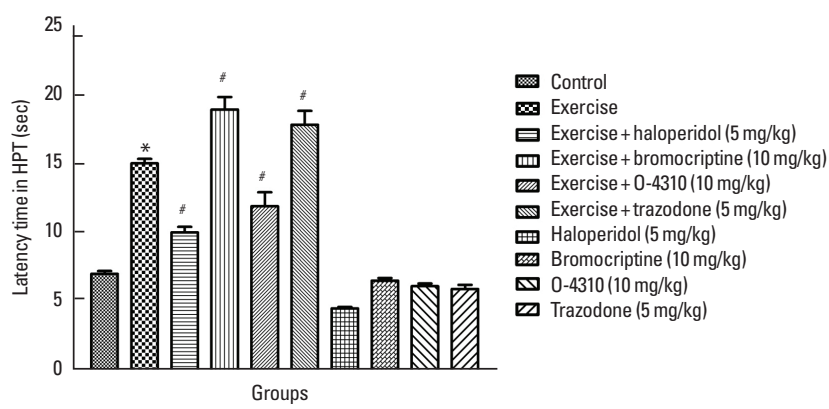

Fig. 4. Latency time of hot plate test (HPT) reaction in the control group and which their mother treated by exercise or its combination with haloperidol, bromocriptine, trazodone or 0-4310 and groups under treatment by haloperidol, bromocriptine, trazodone or 0-4310 alone. Values are presented as mean \pm standard error of the mean $(\mathrm{n}=8) .{ }^{*} P<0.05$, significant difference from control group. ${ }^{\#} P<0.05$, Significant difference from group which their mother were treated by exercise.

tion with haloperidol or O-4310 caused decrease in latency time in comparison with the group whose mothers were treated with the exercise only $(P<0.05)$ (Fig. 3), but treatment of mothers with the forced exercise in combination with bromocrptine or trazodone has not shown any significant differences in comparison to the group which has been treated with exercise only. The maternal treatment with haloperidol or O-4310 caused the decrease of tail flick latency time while the treatment with bromocrptine or trazodone increased the latency time which was not significant in comparison with the control group (Fig. 3).

\section{The effect of maternal exercise and the role of $D_{2}$ and $5-\mathrm{HT}_{2}$ receptors on offsprings' pain response in HPT}

The results of hot plate test (HPT) indicate the effect of maternal forced exercise on responses of offsprings to pain stimulus (Fig.

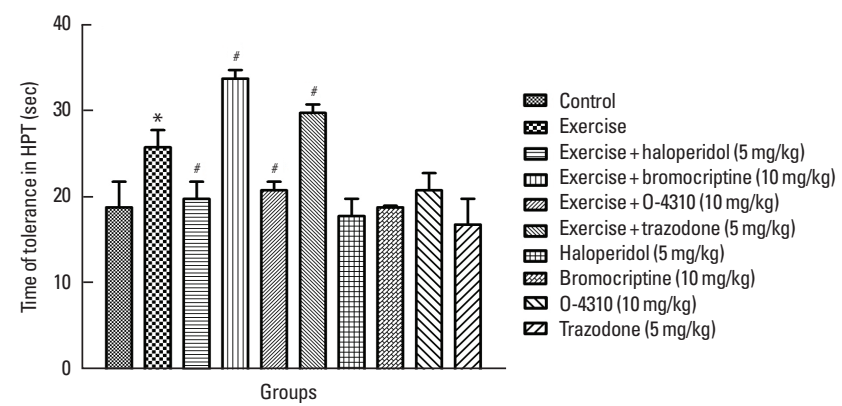

Fig. 5. Hot plate test (HPT) time tolerance in the control group and which their mother treated by exercise or its combination with haloperidol, bromocriptine, trazodone or 0-4310 and groups under treatment by haloperidol, bromocriptine, trazodone or 0-4310 alone. Values are presented as mean \pm standard error of the mean $(n=8)$. ${ }^{*} P<0.05$, significant difference from control group. ${ }^{\#} P<0.05$, Significant difference from group which their mother were treated by exercise.

4). The maternal exercise did not change the latency time of HPT comparing to the control group. However, the treatment of mothers by forced exercise in combination with haloperidol or O-4310 caused the decrease in latency time of HPT and treatments of mothers by forced exercise in combination with bromocrptine or trazodone caused an increase in latency time of HPT, all these changes was statistically significant in comparison with the group whose mothers were treated with the exercise only $(P<0.05)$ (Fig. 4). Also the maternal treatment by haloperidol or O-4310 caused the decrease in latency time of HPT while the treatment with bromocrptine or trazodone increased the latency time in HPT in offsprings, both of these changes were not significant (Fig. 4).

Fig. 5 indicates that the maternal exercise increased the HPT time tolerance in comparison to the control group $(P<0.05)$ (Fig. $5)$. The maternal forced exercise in combination with haloperidol or O-4310 caused a decrease and the combination treatment with bromocriptine or trazodone increased the tolerance in HPT comparing to the group whose mothers were treated with the exercise only $(P<0.05)$ (Fig. 5). In addition, the maternal treatment by haloperidol, O-4310, bropmocriptine or trazodone did not change the time of tolerance in HPT in offsprings as compared to the control group (Fig. 5).

\section{The effect of maternal exercise and the role of $D_{2}$ and $5-\mathrm{HT}_{2}$ receptors on offsprings' OFT behavior assay}

As it is shown in Table 1, the group whose mother were trained with the forced exercise has more frequency of central square entries, more time spent in the central region of the OFT and also more ambulation number and rearing in comparison with the control group $(P<0.05)$ (Table 1$)$. The treatment of mothers with 
Table 1. Effect of maternal exercise and role of $\mathrm{D}_{2}$ and $5-\mathrm{HT}_{2}$ receptors in offspring open field exploratory and anxiety like behavior $(\mathrm{n}=8)$

\begin{tabular}{|c|c|c|c|c|}
\hline Group & Ambulation distance & Time spent in central square & Central square entries & Number of rearing \\
\hline Control & $402 \pm 20$ & $30 \pm 2$ & $8 \pm 2$ & $9 \pm 2$ \\
\hline Exercise & $489 \pm 14^{*}$ & $101 \pm 8^{*}$ & $25 \pm 3^{*}$ & $19 \pm 1^{*}$ \\
\hline Exercise + haloperidol $(5 \mathrm{mg} / \mathrm{kg})$ & $419 \pm 12^{*}$ & $75 \pm 9^{\#}$ & $19 \pm 2^{*}$ & $16 \pm 2^{\#}$ \\
\hline Exercise+bromocriptine $(10 \mathrm{mg} / \mathrm{kg})$ & $518 \pm 11^{\#}$ & $131 \pm 9^{*}$ & $39 \pm 2^{\#}$ & $22 \pm 3^{*}$ \\
\hline Exercise + trazodone $(5 \mathrm{mg} / \mathrm{kg})$ & $436 \pm 15^{\#}$ & $67 \pm 11^{\#}$ & $21 \pm 11^{\#}$ & $17 \pm 3^{\#}$ \\
\hline Exercise + 0-4310 (10 mg/kg) & $500 \pm 10^{*}$ & $115 \pm 10^{\#}$ & $36 \pm 3^{\#}$ & $21 \pm 3^{\#}$ \\
\hline Haloperidol (5 mg/kg) & $385 \pm 19$ & $28 \pm 8$ & $7 \pm 1$ & $9 \pm 3$ \\
\hline Bromocriptine (10 mg/kg) & $410 \pm 13$ & $41 \pm 6$ & $10 \pm 4$ & $11 \pm 4$ \\
\hline $0-4310(10 \mathrm{mg} / \mathrm{kg})$ & $373 \pm 15$ & $20 \pm 3$ & $8 \pm 4$ & $8 \pm 5$ \\
\hline Trazodone (5 mg/kg) & $411 \pm 9$ & $40 \pm 6$ & $9 \pm 2$ & $10 \pm 4$ \\
\hline
\end{tabular}

Values are presented as mean \pm standard error of the mean.

${ }^{*} P<0.05$, significant difference from control group. ${ }^{*} P<0.05$, Significant difference from group which their mother were treated by exercise.

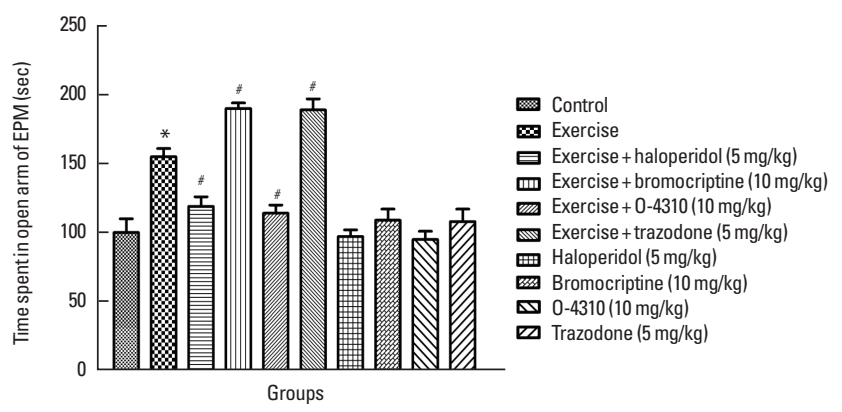

Fig. 6. Duration of time spent in open arms (sec) in elevated plus maze (EPM) test in the control group which their mother treated by exercise or its combination with Haloperidol, bromocriptine, trazodone or 0-4310 and groups under treatment by haloperidol, bromocriptine, trazodone or 0-4310 alone. Values are presented as mean \pm standard error of the mean $(n=8)$. ${ }^{*} P<0.05$, significant difference from control group. ${ }^{\sharp} P<0.05$, Significant difference from group which their mother were treated by exercise.

forced exercise in combination with haloperidol or O-4310 caused the decrease of central square entries, the duration of time spent in the central region and the ambulation number and rearing in comparison with the group whose mothers were treated with exercise only $(P<0.05)$ (Table 1$)$. The treatment of mothers with trazodone or bromocriptine in combination with forced exercise increased the mentioned behavior comparing to the group whose mothers were treated with the exercise only $(P<0.05)$ (Table 1$)$. The maternal treatment with haloperidol or O-4310 decreased the mentioned behavior but bromocriptine or trazodone increased OFT behaviors in offsprings, but these results were not statistically significant (Table 1).

The effect of maternal exercise and the role of $D_{2}$ and $5-\mathrm{HT}_{2}$ receptors on offsprings' anxiety like behavior in EPM

Fig. 6 indicates the effect of maternal forced exercise on EPM in offsprings. The maternal exercise significantly increased the duration spent in open arm in EPM comparing to the control group of offspring $(P<0.05)$ (Fig. 6). Also, the treatment of mothers with forced exercise in combination with haloperidol or O-4310 caused decrease in time spent in open arm in EPM while bromocriptine or trazodone increased this time in comparison to the group whose mothers were treated with exercise only $(P<0.05)$ (Fig. 6). The group whose mothers were treated with haloperidol or O-4310 spent less time in open arm in EPM, and the group whose mother were treated with bromocriptine or trazodone spent more time in open arm in EPM in comparison to the control group $(P<0.05)$ (Fig. 6).

\section{The alteration in CREB gene expression in offsprings}

Maternal exercise significantly increased the expression of CREB in offsprings as compared to the control group $(P<0.05)$ (Fig. 7). Groups whose mother received haloperidol or ketanserin in combination with exercise showed significant decrease in offsprings' CREB gene expression. In contrast, in group whose mothers were treated with bromocriptine or trazodone, the expression of $C R E B$ gene was increased. All these changes were significant in comparison with the offsprings of mothers that were treated only with exercise $(P<0.05)$ (Fig. 7). The maternal treatment by bromocriptine, haloperidol and trazodone caused changes in CREB gene expression in offsprings but these changes were not statistically significant (Fig. 7).

\section{The alteration in cell density and morphology of DG in offsprings}

The maternal exercise significantly increased the number of the granular cells in DG in offsprings' hippocampus as compared to 


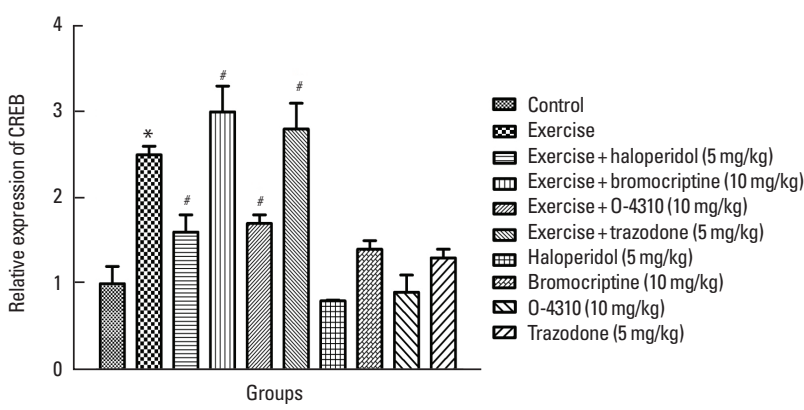

Fig. 7. Alteration of expression of cyclic AMP response element binding protein (CREB) in the control group and groups which their mother treated by exercise or its combination with haloperidol, bromocriptine, trazodone or 0-4310 and groups under treatment by haloperidol, bromocriptine, trazodone or 0-4310 alone. Values are presented as mean \pm standard error of the mean $(\mathrm{n}=8) .{ }^{*} P<0.05$, significant difference from control group. ${ }^{*} P<0.05$, Significant difference from group which their mother were treated by exercise.

Table 2. Effect of maternal exercise and role of $\mathrm{D}_{2}$ and $5-\mathrm{HT}_{2}$ receptors in offspring hippocampus neuronal cell counting (number $/ \mathrm{mm})(\mathrm{n}=8)$

\begin{tabular}{lc}
\hline Group & Neuronal density (number/mm) \\
\hline Control & $502.14 \pm 4.9$ \\
Exercise & $629.11 \pm 5.1^{*}$ \\
Exercise+haloperidol $(5 \mathrm{mg} / \mathrm{kg})$ & $582.0 \pm 9.5^{*}$ \\
Exercise+bromocriptine $(10 \mathrm{mg} / \mathrm{kg})$ & $632.1 \pm 3.4$ \\
Exercise+trazodone $(5 \mathrm{mg} / \mathrm{kg})$ & $624.3 \pm 8.3$ \\
Exercise+0-4310 $(10 \mathrm{mg} / \mathrm{kg})$ & $587.6 \pm 9.1^{*}$ \\
Haloperidol $(5 \mathrm{mg} / \mathrm{kg})$ & $498.5 \pm 7.4$ \\
Bromocriptine $(10 \mathrm{mg} / \mathrm{kg})$ & $485.8 \pm 8.1$ \\
0-4310 $(10 \mathrm{mg} / \mathrm{kg})$ & $491.6 \pm 8.8$ \\
Trazodone $(5 \mathrm{mg} / \mathrm{kg})$ & $481.3 \pm 6.2$ \\
\hline
\end{tabular}

Values are presented as mean \pm standard error of the mean.

${ }^{*} P<0.05$, significant difference from control group. ${ }^{*} P<0.05$, Significant difference from group which their mother were treated by exercise.

the control group $(P<0.05)$ (Table 2$)$. A quantitative comparison of the granular cells in DG density in the group whose mothers was treated with the exercise in combination with haloperidol or O-4310 showed the decrease in granular cells' density in DG in comparison to the group whose mothers were treated with the exercise only $(P<0.05)$ (Table 2$)$. However, the mothers treated with the exercise in combination with bromocriptine or trazodone showed an increase which was not significantly different in comparison to the group whose mothers were treated with the exercise only. The maternal treatment with bromocriptine, haloperidol, trazodone and O-4310 decreased granular cells' density in DG in comparison to the control group and this was not statistically significant (Table 2). As it is shown in Fig. 8, the forced exercise in comparison to the control group caused the increase of DG cell layers. There was a dramatic loss of DG cell layers in group whose

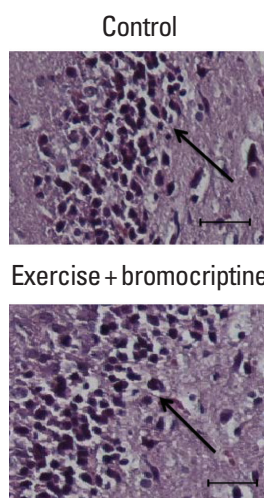

Haloperidol
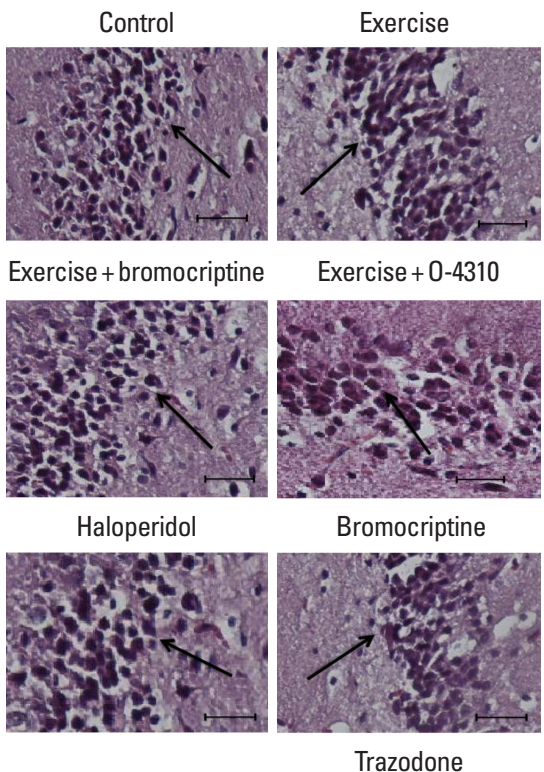

Exercise + haloperidol

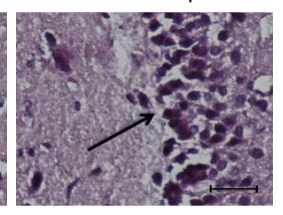

Exercise $+0-4310$

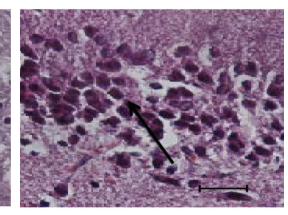

Bromocriptine

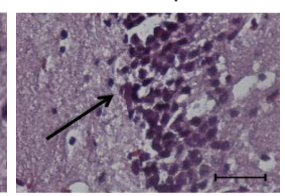

Trazodone

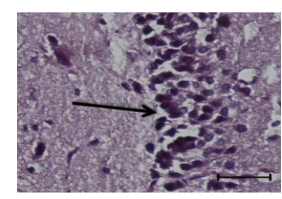

Fig. 8. Hematoxylin and eosin staining of the shows dentate gyrus (DG) cell layer of the hippocampus in the control group and groups which their mother treated by exercise or its combination with haloperidol, bromocriptine, trazodone or 0-4310 and groups under treatment by haloperidol, bromocriptine, trazodone or 0-4310 alone (magnification, $\times 400$ ). Scale bar represents $100 \mu \mathrm{m}$. Arrow shows cell layers in all groups.

mother treated with the exercise in combination with haloperidol or $\mathrm{O}-4310(P<0.05)$, while the exercise in combination with bromocriptine or trazodone did not have any effect on DG cell layers in offsprings. The maternal treatment with bromocriptine, haloperidol, trazodone and O-4310 could change DG cell layers, but this was not statistically significant (Fig. 8).

\section{The correlation between hippocampal CREB expression and gentate gyrus cell density}

The Pearson correlation analysis also demonstrated a significant correlation between hippocampal DG cell density and CREB mRNA expression in all experimental groups $(r=0.9744, P<$ 0.001) (Fig. 9).

\section{DISCUSSION}

The present study indicated that the maternal forced exercise could modulate pain perception, anxiety, depression and CREB gene expression in pups. The maternal forced exercise caused some 


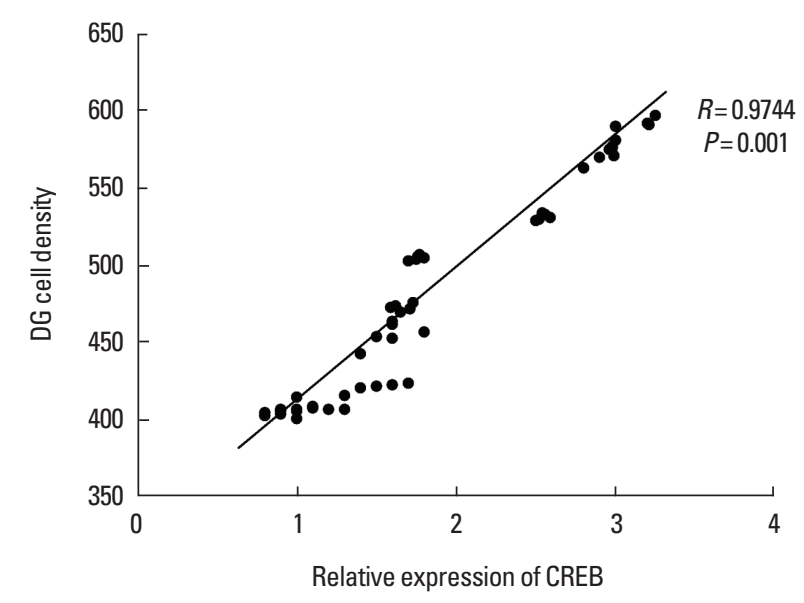

Fig. 9. Correlation analysis between the cyclic AMP response element binding protein (CREB) expression and dentate gyrus (DG) cell density in all groups. $r=0.9744, P<0.001$.

behavioral alterations in FST (swimming), EPM (open arm entry), and OFT (central area entry, central area duration, ambulation, and rearing) of their offsprings. Moreover, the maternal forced exercise could diminish the pain perception in pups, and that probably was modulated with $\mathrm{D}_{2}$ (dopaminergic) receptor and 5- $\mathrm{HT}_{1}$ (serotonergic) receptor which consequently caused the alteration of $C R E B$ gene expression level. Previous studies have revealed that physical activity lowers stress and anxiety levels and releases endorphins into the brain. Exercise can counteract drug abuse withdrawal symptoms with the attenuation of depression, the reduction of anxiety and help the patient to feel better. Several research studies have demonstrated that exercise can manage this recovery process. Physical activity can increase the synthesis and the release of dopamine, stimulate neuroplasticity and promote the feelings of well-being. Chronic exercise in mice results in antidepressant-like behavioral changes that may involve a BDNF related mechanism similar to the hypothesis for antidepressant drug treatment (Aksu et al., 2012; Lee et al., 2006).

Also the present study showed that the maternal forced exercise decreased the number of writhings in offsprings. This study showed that the effect was modulated by dopamine $\mathrm{D}_{2}$ and serotonin 5-HT $\mathrm{H}_{2}$ receptors. The treatment with haloperidol or ketanserin inhibited the protective effect of maternal forced exercise on the abdominal pain in writhing test. Treatment of mothers with forced exercise, trazodone and bromocriptine enabled the protective effects of forced exercise on offsprings pain management. Many previous studies demonstrated that the maternal physical activity could develop the neural regeneration in offsprings (Herring et al., 2012) and based on the present study, one kind of neu- ral development was the pain perception and the increase in pain tolerability (Galdino et al., 2010). On the other hand, previous researches have shown that $\mathrm{D}_{2}$ and $5-\mathrm{HT}_{2}$ receptors can modulate the pain and these receptors play an important role in neuronal regeneration (Borta and Höglinger, 2007; Whitaker-Azmitia, 2001). They have also shown that $\mathrm{D}_{2}$ and $5-\mathrm{HT}_{2}$ agonists have analgesic effect suggesting that the receptors can modulate pain perception pathways in brain. Based on this concept we can argue that forced exercise have effect on offsprings' pain tolerability probably through $\mathrm{D}_{2}$ and $5-\mathrm{HT}_{2}$ receptors (Treister et al., 2011; Wood, 2008).

Offsprings of mothers that were treated with haloperidol considerably increased the writhing number in comparison to the control group but the treatment with bromocrptine or trazodone significantly decreased the writhing number in comparison to the control group. The decrease was significant in trazodone treated group. The results can be discussed on the basis of previous studies which showed that bromocrptine or trazodone has neuroprotective effect and this probably could be the reason for brain development (Jensen et al., 2008; Huey et al., 2006).

The present study showed that maternal forced exercise increases the latency time in writhing test in offsprings and the treatment of mothers by haloperidol in combination with forced exercise decreases the latency time in writhing test. Moreover, in mothers treated with forced exercise and bromocrptine or trazodone, the latency time was increased in writhing test. Physical activity could cause inhibition of inflammatory and neuropathic pain in offsprings, previous studies have shown that physical activity in mothers cause modulation of neurohormone and potentiate immunological function in children and attenuates immunologic disorder in offsprings (Gleeson, 2007; Vega et al., 2015). It was approved that $\mathrm{D}_{2}$ and $5-\mathrm{HT}_{2}$ receptors have important role in inflammatory pain. Also, agonists on these receptors can inhibit the expression of inflammatory and acetic acid induced pain in animal models (Shojaii et al., 2015; Zakaria et al., 2005). In present study, pups whose mothers were treated with haloperidol, trazodone caused decrease in latency time while in pups whose mother treated with bromocrptine caused the increase of latency time. Based on this study, there might be a link between maternal forced exercise and $\mathrm{D}_{2}$ and $5-\mathrm{HT}_{2}$ receptors' involvement in brain pathways of inflammatory pain perception.

In TFT, for evaluation of maternal forced exercise on spinal pain perception, this research showed that forced exercise in mothers caused an increase in latency time of TFT and this effect was probably mediated through $5-\mathrm{HT}_{2}$ and $\mathrm{D}_{2}$ receptors. The results 
showed forced exercise in combination with haloperidol caused the decrease in the latency time in comparison to the group whose mothers were treated with exercise only.

Many previous studies showed that physical activity increase the tolerance against pain stimulant especially in spinal pain. Moreover, there is growing number of evidence supporting the role of dopamine and serotonin in spinal pain perception (Berger et al., 2009; Potvin et al., 2009; Treister et al., 2011). The present study showed that protective role of forced exercise in management of spinal pain perception in tail flick was mediated by dopamine and serotonin in which $5-\mathrm{HT}_{2}$ and $\mathrm{D}_{2}$ receptors were involved.

This research indicated that maternal forced exercise might cause increase of the hot plate tolerance time in offsprings. On the other hand, the present study suggested that this effect might be mediated by dopamine $\mathrm{D}_{2}$ receptor and serotonin $5-\mathrm{HT}_{2}$ receptor since treatment by haloperidol inhibited the effect of maternal forced exercise and abolished the tolerance. Also, treatment of mothers by bromocriptine and trazodone increased the tolerance of offsprings to pain stimulus in HPT. The maternal treatment by haloperidol decreased the duration of tolerance and treatment with bropmocriptine or trazodone increased the time of tolerance in the HPT in offsprings in comparison to the control group. Accordingly, in HPT the latency time in offspring of mothers that were treated with forced exercise only were increased while haloperidol could inhibit this effect and trazodone or bromocrptine potentiated the effect of forced exercise. The mother's treatment with ketanserin or haloperidol also decreased the latency time and trazodone or bromcriptine increased it in pups. About the effect of forced exercise in pain management similar to other researches, this study indicated that maternal activity could potentiate offspring tolerance and endogenous analgesic pathways in HPT model. Many previous studies have demonstrated that maternal physical activity can increase the number of receptors for bioamines in brain and spinal cord, so this increase could be responsible for various protective effects of physical activity in brain disorders (Foley and Fleshner, 2008; Lan et al., 2014; Ma et al., 2008). This research confirmed the concept which maternal forced exercise had effects on the pain perception of pups in HPT was and this probably is mediated by $\mathrm{D}_{2}$ and $5-\mathrm{HT}_{2}$ receptors and it could show the role of serotonin and dopamine in forced exercise neuroprotection in pain perception pathway synaptic plasticity.

In OFT, This research indicated that pups of mothers treated with forced exercise had more frequency of central square entries, and spent more time in the central region of the OFT, more ambulation and rearing number in this test. Treatment of mothers by haloperidol in combination with forced exercise abolished the effect of forced exercise on this behavior which suggests that antidepressant and anxiolytic effect of exercise might have been mediated by $\mathrm{D}_{2}$ and/or $5-\mathrm{HT}_{2}$ receptors. Treatment of mothers by trazodone or bromocriptine in combination with forced exercise increased the frequency of central square entries, time spent in the central region, ambulation and rearing number in pups. Pups whose mothers were treated with haloperidol showed attenuation and those whom their mothers were treated with bromocriptine or trazodone showed improvement in OFT behaviors in comparison to the control group. Forced exercise in mothers can modulate motor activity in mothers as well as pups (Choi et al., 2013; Dayi et al., 2012). Many previous studies demonstrated that deficiency in dopamine and serotonin cause motor dysfunction and the present study confirmed this concept. Blockade of $\mathrm{D}_{2}$ or $5-\mathrm{HT}_{2}$ receptors in mothers causes motor activity dysfunction in offsprings which is shown in OFT (Robinson and Bucci, 2012). On the other hand, forced exercise in mothers has neuroprotective effect and potentiates the plasticity of neurons which is responsible for motor activity (Robinson and Bucci, 2012). $\mathrm{D}_{2}$ or $5-\mathrm{HT}_{2}$ receptors probably mediate the effect of forced exercise since their antagonist inhibits this effect of forced exercise while their agonist has potentiated the effect in OFT. The maternal forced exercise causes increase in time spent by pups in open arm in EPM as compared to the control group in offsprings. On the other hand, haloperidol inhibited this effect of forced exercise, but bromocriptine and trazodone potentiate the anxiolytic effect of exercise and increase the time spent in open arm in EPM. Also, treatment of mothers by bromocriptine, trazodone and haloperidol cause changes in anxiety like behavior in open arm in EPM in their offsprings. Many studies have shown that depressive and anxiety like behaviors in offsprings of mothers who had a regular exercise during their pregnancy is less than the pups whose mothers had no physical activity during their pregnancy (Akhavan et al., 2008; Mello et al., 2009; Uysal et al., 2011). This study confirmed this concept and indicated that anxiolytic and antidepressant effect of maternal exercise on offsprings were mediated by $\mathrm{D}_{2}$ or $5-\mathrm{HT}_{2}$ receptors which are probably involved in protective effect of maternal forced exercise.

Maternal forced exercise increases the CREB gene expression as compared to the control group in offsprings. On the other hand, haloperidol inhibits this effect of forced exercise, but bromocriptine and trazodone potentiate the effect of maternal forced exercise on $C R E B$ gene expression in pups. Also, treatment of mothers with bromocriptine or trazodone increase $C R E B$ gene expression while haloperidol or ketanserin decrease it in offsprings. Many 
previous studies have indicated that CREB as transcription factor has main protective roles against some neurodegenerative conditions such as Parkinson, Alzheimer, and seizure. On the other hand, many studies have demonstrated that physical activity can increase gene expression and protect the brain against oxidative agents (Barco and Marie, 2011; Saura and Valero, 2011). This research suggested that maternal forced exercise increased CREB gene expression and probably its protective effect in offspring such as anxiolytic, antidepressant and tolerability to pain were mediated by increase in $C R E B$ gene expression in pups. Also, the present study showed that the effect of forced exercise in CREB gene expression was mediated by $\mathrm{D}_{2}$ or $5-\mathrm{HT}_{2}$ receptors, in which combination treatment of mothers by forced exercise with $\mathrm{D}_{2}$ or 5- $\mathrm{HT}_{2}$ receptor agonists potentiated this transcription factor expression while $\mathrm{D}_{2}$ or $5-\mathrm{HT}_{2}$ receptor antagonists abolished the effects of forced exercise on offsprings. This study indicated that maternal forced exercise caused the increase of cell density in DG region of hippocampus in offsprings and forced exercise increased DG cell layer in children. Previous study have shown that maternal exercise caused development of offsprings brain especially hippocampus (Kim et al., 2007).The treatment of mothers with forced exercise in combination with haloperidol or ketanserin, decreased the cell density and caused dramatic loss in cell layer of DG and suggest that $\mathrm{D}_{2}$ or $5-\mathrm{HT}_{2}$ receptor has significant role in protective effect of maternal forced exercise on brain cell density. Many previous studies have demonstrated that $\mathrm{D}_{2}$ or $5-\mathrm{HT}_{2}$ receptor has important role in brain development during growth in mothers' uterus (Akhavan et al., 2008; Mamo et al., 2004) Histomorphological findings of the present research suggested that some part of protective maternal physical activity was mediated by $\mathrm{D}_{2}$ or $5-\mathrm{HT}_{2}$ receptors. Also this study showed that there was correlation between cell density in DG region and CREB gene expression. It suggested that there was a great correlation between maternal forced exercise and CREB gene expression, as one of main transcription factors involved in neuroprotection in brain (Oury et al., 2010).The findings of this correlation between forced exercises induced elevation in cell density in DG region and neurodevelopment is a novel achievement. This research confirmed previous results showed that CREB has strategic role in many degenerative diseases such as neurodegenerative disorders with defect in expression of this transcription factor (Li et al., 2009). The data obtained in present study supported the hypothesis that the maternal physical activity has neuroprotective properties in offsprings such as reduction of anxiety and depression and increase in pain tolerability. Also, this research showed that these neuropro- tective effects of maternal forced exercise might probably be mediated by $\mathrm{D}_{2}$ and/or $5-\mathrm{HT}_{2}$ receptors and CREB gene expression as main downstream transcription factor of these receptors.

\section{CONFLICT OF INTEREST}

No potential conflict of interest relevant to this article was reported.

\section{REFERENCES}

Akhavan MM, Emami-Abarghoie M, Safari M, Sadighi-Moghaddam B, Vafaei AA, Bandegi AR, Rashidy-Pour A. Serotonergic and noradrenergic lesions suppress the enhancing effect of maternal exercise during pregnancy on learning and memory in rat pups. Neuroscience 2008; 151:1173-1183.

Aksu I, Baykara B, Ozbal S, Cetin F, Sisman AR, Dayi A, Gencoglu C, Tas A, Büyük E, Gonenc-Arda S, Uysal N. Maternal treadmill exercise during pregnancy decreases anxiety and increases prefrontal cortex VEGF and BDNF levels of rat pups in early and late periods of life. Neurosci Lett 2012;516:221-225.

Barco A, Marie H. Genetic approaches to investigate the role of CREB in neuronal plasticity and memory. Mol Neurobiol 2011;44:330-349.

Benninghoff J, van der Ven A, Schloesser RJ, Moessner R, Möller HJ, Rujescu D. The complex role of the serotonin transporter in adult neurogenesis and neuroplasticity. A critical review. World J Biol Psychiatry 2012;13:240-247.

Berger M, Gray JA, Roth BL. The expanded biology of serotonin. Annu Rev Med 2009;60:355-366.

Borta A, Höglinger GU. Dopamine and adult neurogenesis. J Neurochem 2007;100:587-595.

Cho HS, Shin MS, Song W, Jun TW, Lim BV, Kim YP, Kim CJ. Treadmill exercise alleviates short-term memory impairment in 6-hydroxydopamine-induced Parkinson's rats. J Exerc Rehabil 2013;9:354-361.

Choi JH, Kim TS, Park JK, Sim YJ, Kim K, Lee SJ. Short-term treadmill exercise preserves sensory-motor function through inhibiting apoptosis in the hippocampus of hypoxic ischemia injury rat pups. J Exerc Rehabil 2013;9:457-462.

Cotman CW, Berchtold NC. Exercise: a behavioral intervention to enhance brain health and plasticity. Trends Neurosci 2002;25:295-301.

Dayi A, Agilkaya S, Ozbal S, Cetin F, Aksu I, Gencoglu C, Cingoz S, Pekcetin C, Tugyan K, Kayatekin BM, Uysal N. Maternal aerobic exercise during pregnancy can increase spatial learning by affecting leptin expression on offspring's early and late period in life depending on gender. ScientificWorldJournal 2012;2012:429803. 
Dorak MT. Real-time PCR. Oxford: Taylor \& Francis; 2007.

Dworkin S, Mantamadiotis T. Targeting CREB signalling in neurogenesis. Expert Opin Ther Targets 2010;14:869-879.

Foley TE, Fleshner M. Neuroplasticity of dopamine circuits after exercise: implications for central fatigue. Neuromolecular Med 2008;10:67-80.

Galdino GS, Duarte ID, Perez AC. Participation of endogenous opioids in the antinociception induced by resistance exercise in rats. Braz J Med Biol Res 2010;43:906-909.

Gaspar P, Cases O, Maroteaux L. The developmental role of serotonin: news from mouse molecular genetics. Nat Rev Neurosci 2003;4:10021012.

Gass P, Riva MA. CREB, neurogenesis and depression. Bioessays 2007; 29:957-961.

Gleeson M. Immune function in sport and exercise. J Appl Physiol (1985) 2007;103:693-699.

Herring A, Donath A, Yarmolenko M, Uslar E, Conzen C, Kanakis D, Bosma C, Worm K, Paulus W, Keyvani K. Exercise during pregnancy mitigates Alzheimer-like pathology in mouse offspring. FASEB J 2012; 26:117-128.

Hosseinzadeh S, Zahmatkesh M, Heidari M, Hassanzadeh GR, Karimian M, Sarrafnejad A, Zarrindast MR. Hippocampal DHCR24 down regulation in a rat model of streptozotocin-induced cognitive decline. Neurosci Lett 2015;587:107-112.

Huey ED, Putnam KT, Grafman J. A systematic review of neurotransmitter deficits and treatments in frontotemporal dementia. Neurology 2006;66:17-22.

Jensen RV, Gullans SR, Sarang SS, Yoshida T, Cadet R, Valeras AS. Discovery of molecular mechanisms of neuroprotection. Neurosci 2008; 28:9473-9485.

Kim BK, Seo JH. Treadmill exercise alleviates post-traumatic stress disorder-induced impairment of spatial learning memory in rats. J Exerc Rehabil 2013;9:413-419.

Kim CJ. New horizon for the therapeutic modality of exercise on neuropsychiatric disorders. J Exerc Rehabil 2013;9:449-450.

Kim H, Lee SH, Kim SS, Yoo JH, Kim CJ. The influence of maternal treadmill running during pregnancy on short-term memory and hippocampal cell survival in rat pups. Int J Dev Neurosci 2007;25:243-249.

Kim YM, Seo TB, Kim CJ, Ji ES. Treadmill exercise with bone marrow stromal cells transplantation potentiates recovery of locomotor function after spinal cord injury in rats. J Exerc Rehabil 2017;13:273-278.

Kramer AF, Erickson KI, Colcombe SJ. Exercise, cognition, and the aging brain. J Appl Physiol (1985) 2006;101:1237-1242.

Kumar SK, Perumal S, Rajagopalan V. Therapeutic effect of bone marrow mesenchymal stem cells on cold stress induced changes in the hippocampus of rats. Neural Regen Res 2014;9:1740-1744.
Lan X, Zhang M, Yang W, Zheng Z, Wu Y, Zeng Q, Liu S, Liu K, Li G. Effect of treadmill exercise on 5-HT, 5-HT1A receptor and brain derived neurophic factor in rats after permanent middle cerebral artery occlusion. Neurol Sci 2014;35:761-766.

Lawlor DA, Hopker SW. The effectiveness of exercise as an intervention in the management of depression: systematic review and meta-regression analysis of randomised controlled trials. BMJ 2001;322:763-767.

Lee HH, Kim H, Lee JW, Kim YS, Yang HY, Chang HK, Lee TH, Shin MC, Lee MH, Shin MS, Park S, Baek S, Kim CJ. Maternal swimming during pregnancy enhances short-term memory and neurogenesis in the hippocampus of rat pups. Brain Dev 2006;28:147-154.

Li S, Zhang C, Takemori H, Zhou Y, Xiong ZQ. TORC1 regulates activity-dependent CREB-target gene transcription and dendritic growth of developing cortical neurons. J Neurosci 2009;29:2334-2343.

Liu Y, Wang PS, Xie D, Liu K, Chen L. Ghrelin reduces injury of hippocampal neurons in a rat model of cerebral ischemia/reperfusion. Chin J Physiol 2006;49:244-250.

Ma Q, Wang J, Chen XW, An GH, Liu HT. Alterations in rat hippocampal norepinephrine and serotonin levels under physical exercise and psychological stress. Chin J Physiol 2008;8:1549-1552.

Mamo D, Kapur S, Shammi CM, Papatheodorou G, Mann S, Therrien F, Remington G. A PET study of dopamine D2 and serotonin 5-HT2 receptor occupancy in patients with schizophrenia treated with therapeutic doses of ziprasidone. Am J Psychiatry 2004;161:818-825.

Mello PB, Benetti F, Cammarota M, Izquierdo I. Physical exercise can reverse the deficit in fear memory induced by maternal deprivation. Neurobiol Learn Mem 2009;92:364-369.

Merz K, Herold S, Lie DC. CREB in adult neurogenesis--master and partner in the development of adult-born neurons? Eur J Neurosci 2011; 33:1078-1086.

Motaghinejad M, Bangash MY, Hosseini P, Karimian SM, Motaghinejad O. Attenuation of morphine withdrawal syndrome by various dosages of curcumin in comparison with clonidine in mouse: possible mechanism. Iran J Med Sci 2015a;40:125-132.

Motaghinejad M, Ghaleni MA, Motaghinejad O. Preventive effects of forced exercise against alcohol-induced physical dependency and reduction of pain perception threshold. Int J Prev Med 2014a;5:12991307.

Motaghinejad M, Motevalian M, Asadi-Ghalehni M, Motaghinejad O. Attenuation of morphine withdrawal signs, blood cortisol and glucose level with forced exercise in comparison with clonidine. Adv Biomed Res 2014b;3:171.

Motaghinejad M, Motevalian M, Ebrahimzadeh A, Larijani SF, Khajehamedi Z. Reduction of methylphenidate induced anxiety, depression and cognition impairment by various doses of venlafaxine in rat. Int J 
Prev Med 2015b;6:52.

Motaghinejad M, Motevalian M, Larijani SF, Khajehamedi Z. Protective effects of forced exercise against methylphenidate-induced anxiety, depression and cognition impairment in rat. Adv Biomed Res 2015c; 4:134.

Noori N, Bangash MY, Motaghinejad M, Hosseini P, Noudoost B. Kefir protective effects against nicotine cessation-induced anxiety and cognition impairments in rats. Adv Biomed Res 2014;3:251.

Ojoawo AO, Hassan MA, Olaogun MOB, Johnson EO, Mbada CE. Comparative effectiveness of two stabilization exercise positions on pain and functional disability of patients with low back pain. J Exerc Rehabil 2017;13:363-371.

Oury F, Yadav VK, Wang Y, Zhou B, Liu XS, Guo XE, Tecott LH, Schutz G, Means AR, Karsenty G. CREB mediates brain serotonin regulation of bone mass through its expression in ventromedial hypothalamic neurons. Genes Dev 2010;24:2330-2342.

Park JK, Lee SJ, Oh CS. Treadmill exercise exerts ameliorating effect on isolation-induced depression via neuronal activation. J Exerc Rehabil 2013;9:234-242.

Potvin S, Grignon S, Marchand S. Human evidence of a supra-spinal modulating role of dopamine on pain perception. Synapse 2009;63: 390-402.

Robinson AM, Bucci DJ. Maternal exercise and cognitive functions of the offspring. Cogn Sci (Hauppauge) 2012;7:187-205.

Salmon P. Effects of physical exercise on anxiety, depression, and sensitivity to stress: a unifying theory. Clin Psychol Rev 2001;21:33-61.

Saura CA, Valero J. The role of CREB signaling in Alzheimer's disease and other cognitive disorders. Rev Neurosci 2011;22:153-169.

Shojaii A, Motaghinejad M, Norouzi S, Motevalian M. Evaluation of anti-inflammatory and analgesic activity of the extract and fractions of astragalus hamosus in animal models. Iran J Pharm Res 2015;14:263269.

Tomporowski PD. Effects of acute bouts of exercise on cognition. Acta Psychol (Amst) 2003;112:297-324.

Treister R, Pud D, Ebstein RP, Laiba E, Raz Y, Gershon E, Haddad M, Eisenberg E. Association between polymorphisms in serotonin and dopamine-related genes and endogenous pain modulation. J Pain 2011;12:875-883.

Uysal N, Sisman AR, Dayi A, Aksu I, Cetin F, Gencoglu C, Tas A, Buyuk E. Maternal exercise decreases maternal deprivation induced anxiety of pups and correlates to increased prefrontal cortex BDNF and VEGF. Neurosci Lett 2011;505:273-278.

Vega CC, Reyes-Castro LA, Bautista CJ, Larrea F, Nathanielsz PW, Zambrano $E$. Exercise in obese female rats has beneficial effects on maternal and male and female offspring metabolism. Int J Obes (Lond) 2015;39:712-719.

Whitaker-Azmitia PM. Serotonin and brain development: role in human developmental diseases. Brain Res Bull 2001;56:479-485.

Wood PB. Role of central dopamine in pain and analgesia. Expert Rev Neurother 2008;8:781-797.

Zakaria ZA, Sulaiman MR, Mat Jais AM, Somchit MN. Effect of various antagonists on the Channa striatus fillet extract antinociception in mice. Can J Physiol Pharmacol 2005;83:635-642. 